\title{
Valuation of stress patterns in the peri implant bone of non-parallel implants supporting a long- cantilevered prosthesis: a 3D finite element analysis
}

\author{
Mohammed Abusaad Siddiqui', Sudheer N², Dulala Vikram Raj ${ }^{3}$, Aditi Chintamani Sabnis ${ }^{4}$, \\ Alluru Amrutesh ${ }^{5}$, Anurag Lahoti ${ }^{5}$

\begin{abstract}
Reader, Department of Prosthodontics, Sri Balaji Dental College, Hyderabad, Telangana, India
Professor, Department of prosthodontics, Kamineni Institute of Dental Sciences, Narketpally, Telangana, India

Professor and HOD, Department of Prosthodontics, Sri Balaji Dental College, Hyderabad, Telangana, India

Reader, Department of Prosthodontics, Sri Balaji Dental College, Hyderabad, Telangana, India

Senior Lecturer, Department of Prosthodontics, Sri Balaji Dental College, Hyderabad, Telangana, India
\end{abstract}

\begin{abstract}
The treatment modality for completely edentulous arches has shifted from complete dentures to dental implants during the last 15-20 years. Tilting of implants has reduced the concern of resorbed posterior ridges in completely edentulous patients with "All-on-four" and "All-on-six" concept of dental implants. The purpose of this study is to compare the biomechanical behaviour of the "All-on-four", "All-on-six" models with tilted distal implants at different angulations of 30 and $45^{\circ}$ with four parallel placed implant-supported fixed prosthesis, and six parallel placed implant-supported fixed prosthesis models as controls using three-dimensional finite element analysis. The results showed that in all the models, in cancellous bone, cortical bone, implant and prosthesis - "All-on-four" model with distal implants tilted at an angulation of $30^{\circ}$ showed stress values less than or equivalent to all the other models except on the implant in the presence of cantilever and on prosthesis during full mouth biting load where maximum stresses were observed. The study shows that All-on-four concept with tilted distal implants at an angulation of $30^{\circ}$ showed stress values favourable for the rehabilitation of completely edentulous maxilla, but the presence of cantilever remains an area of concern.
\end{abstract}

KEYWORDS: Peri Implant Bone, Non Parallel Imnplants, Cantilevered Prothesis, Stress Patterns

\section{INTRODUCTION}

$\mathrm{D}$ ental implants traditionally have been placed axially in ridges with good quality of bone along the long axis of the desired tooth to accept forces axially down the implant. In completely edentulous jaw, there is often insufficient vertical height after bony resorption in the posterior regions. In the maxilla, sinus augmentation with a lateral window approach is necessary with an extended treatment time of up to 5 months. In the mandible, bone augmentation with only grafts or nerve transposition must be carried out to allow placement of implants posteriorly [1, 2]. Tilting of posterior implants can be carried out for these ridges by taking maximum advantage of existing bone and avoiding the placement of additional implants adding significant time, morbidity, and cost to the procedures [1-3]. The "All-on-4" treatment concept was developed by Paulo Malo and encompassed an immediately loaded full-arch fixed prosthesis anchored with four implants in either the maxilla or mandible. The All on four concepts with straight and angled multi-unit abutments was developed to provide edentulous patients with an immediately loaded full-arch restoration with only four implants-two

Correspondence: Dr. Mohammed Abusaad Siddiqui, Department of Prosthodontics, Sri Balaji Dental College, Hyderabad, Telangana. Email: drabusaadmds@gmail.com

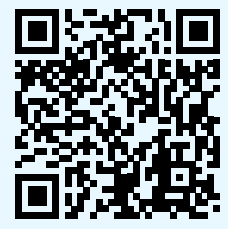


placed vertically in the anterior region and two placed at an angle of up to $45^{\circ}$ in the posterior region 1,2 . The "All-on- 6 " treatment concept of dental implants is a variation of the "All-on-4" technique based on the same concept, with the only difference that four implants are placed vertically in the anterior region and two placed at an angle of up to $45^{\circ}$ in the posterior region. But, because of the biomechanical complexities of dental implants, more detailed information on biomechanical aspects of implants is required if long term success is to be achieved.

\section{Material And Methodology}

The Precise 3D geometric FEM models were simulated. Ideal conditions were established, such as $100 \%$ contact between bone and implant and perfect fit of implants, abutments, and prosthetic bars, absence of gaps or frictional coefficient were verified. The masticatory forces selected for the present study were based on averages found in the literature for patients with implant-supported prostheses.

Six models were fabricated simulating implants placed in a type 3 bone block of the maxilla, assuming that all materials in finite element analysis are homogenous, isotropic, and linearly elastic.

These models were mentioned as: -

Model 1: Four parallel placed - implantsupported cantilevered maxillary prosthesis

Model 2: Six parallel placed- implant-supported cantilevered maxillary prosthesis

Model 3: Four-implant-supported maxillary prosthesis with tilted distal implants at an angle of $30^{\circ}$, from the vertical axis

Model 4: Four-implant-supported maxillary prosthesis with distal implants tilted at an angle of $45^{\circ}$ from the vertical axis

Model 5: Six-implant-supported maxillary prosthesis with tilted distal implants at an angle of $30^{\circ}$ from the vertical axis

Model 6: Six-implant-supported maxillary prosthesis with tilted distal implants at an angle of $45^{\circ}$ from the vertical axis.

These models were then subjected to the following loading conditions: -

Loading condition 1: Full mouth biting load bilateral and simultaneous vertical static load

Loading condition 2: Lateral load - unilateral horizontal static load

Loading condition 3: Presence of cantilever unilateral vertical static load

Loading condition 4: Absence of cantilever load - unilateral vertical static load

The von Mises stresses were obtained for all the six models under the desired loading criteria for cancellous bone, cortical bone, implant, and prosthesis in the present study.

Table 1. Young's moduli and Poisson ratios of the materials used in the present study

\begin{tabular}{|l|l|l|}
\hline Material & $\begin{array}{l}\text { Young's } \\
\text { modulus }\end{array}$ & Poisson's ratio \\
\hline Cortical bone & 13.7 & 0.30 \\
\hline Cancellous bone & 1.37 & 0.30 \\
\hline Titanium & 115 & 0.35 \\
\hline Type III Gold & 100 & 0.30 \\
\hline
\end{tabular}

Table 2. The number of nodes and elements arranged in all the models

\begin{tabular}{|l|l|l|}
\hline Model & Nodes & Elements \\
\hline Model 1 & 143486 & 79739 \\
\hline Model 2 & 146233 & 80829 \\
\hline Model 3 & 144069 & 79957 \\
\hline Model 4 & 146529 & 81562 \\
\hline Model 5 & 147176 & 81262 \\
\hline Model 6 & 149253 & 82622 \\
\hline
\end{tabular}

\section{RESUlts}

In all the models, in cancellous bone, cortical bone, implant and prosthesis - "All-on-four" model with distal implants tilted at an angulation of $30^{\circ}$ showed stress values less than or equivalent to all the other models, i.e. All-onfour model with distal implants tilted at an angulation of $45^{\circ}$; "All-on-six" models with distal implants tilted at an angulation of $30^{\circ}, 45^{\circ}$; four parallelly placed implant model and six parallel placed implant model except in implant (loading criteria three the presence of cantilever) and prosthesis (loading criteria 1 - full mouth biting load) where maximum stresses were observed.

Table 3. The von Mises stresses obtained for all models under the desired loading criteria for cancellous bone

\begin{tabular}{|l|l|l|l|l|l|l|}
\hline \multirow{2}{*}{$\begin{array}{l}\text { Loading } \\
\text { condi } \\
\text { tion }\end{array}$} & \multicolumn{4}{|l|}{ Model } \\
\cline { 2 - 7 } & 1 & 2 & 3 & 4 & 5 & 6 \\
\hline 1 & 17.9 & 21.1 & 9.4 & 14.1 & 10.1 & 16 \\
\hline 2 & 13.1 & 11.8 & 5.2 & 7.4 & 5.3 & 8.4 \\
\hline 3 & 12.4 & 12.8 & 12.3 & 9.7 & 10.5 & 9.1 \\
\hline 4 & 11.9 & 12.1 & 9.5 & 8.3 & 7.8 & 8.7 \\
\hline
\end{tabular}


Table 4. The von Mises stresses obtained for all models under the desired loading criteria for cortical bone

\begin{tabular}{|c|c|c|c|c|c|c|}
\hline \multirow{2}{*}{$\begin{array}{l}\text { Loading } \\
\text { condi- } \\
\text { tion } \\
\end{array}$} & \multicolumn{6}{|c|}{ Model } \\
\hline & 1 & 2 & 3 & 4 & 5 & 6 \\
\hline 1 & 27.6 & 33.4 & 37.9 & 90 & 41 & 100 \\
\hline 2 & 55.8 & 56.3 & 19 & 57 & 20 & 59.8 \\
\hline 3 & 36.3 & 27.7 & 40 & 85 & 40 & 85 \\
\hline 4 & 35.4 & 24.8 & 37.8 & 55 & 31 & 58.8 \\
\hline
\end{tabular}

Table 5. The von Mises stresses obtained for all models under the desired loading criteria for implant

\begin{tabular}{|l|l|l|l|l|l|l|}
\hline \multirow{2}{*}{$\begin{array}{l}\text { Loading } \\
\text { condi } \\
\text { tion }\end{array}$} & \multicolumn{6}{|l|}{ Model } \\
\cline { 2 - 7 } & 1 & 2 & 3 & 4 & 5 & 6 \\
\hline 1 & 120 & 120 & 120 & 90 & 120 & 100 \\
\hline 2 & 20.6 & 23.2 & 31 & 43.7 & 27.4 & 43.4 \\
\hline 3 & 80 & 80 & 100 & 92 & 80 & 92 \\
\hline 4 & 68.5 & 68.5 & 45 & 72.1 & 67.8 & 77.5 \\
\hline
\end{tabular}

Table 6. The von Mises stresses obtained for all models under the desired loading criteria for prosthesis

\begin{tabular}{|l|l|l|l|l|l|l|}
\hline \multirow{2}{*}{$\begin{array}{l}\text { Loading } \\
\text { condi } \\
\text { tion }\end{array}$} & \multicolumn{3}{|l|}{ Model } \\
\cline { 2 - 7 } & 1 & 2 & 3 & 4 & 5 & 6 \\
\hline 1 & 80 & 80 & 100 & 85 & 89 & 82 \\
\hline 2 & 17 & 16 & 17 & 17 & 19 & 15 \\
\hline 3 & 76 & 70 & 80 & 70 & 87 & 70 \\
\hline 4 & 26 & 24 & 22 & 22 & 20 & 28 \\
\hline
\end{tabular}

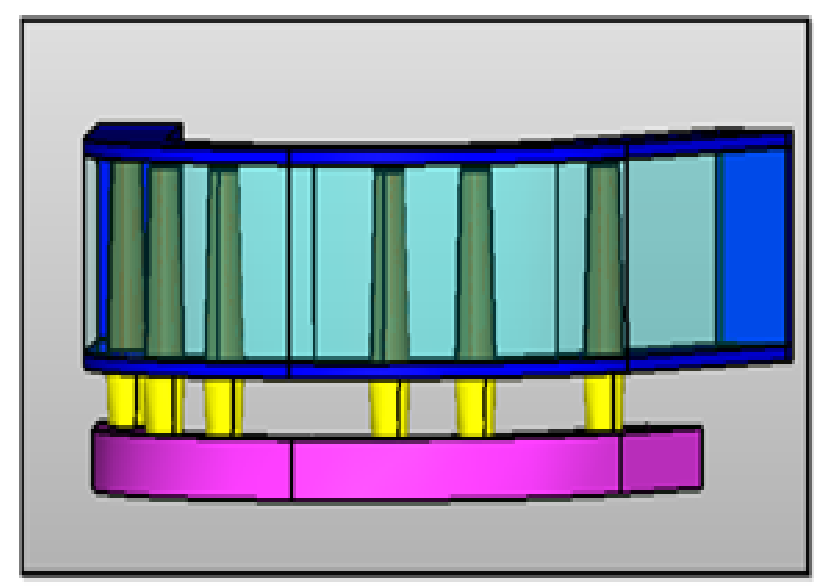

Fig 1. 3D Finite element model of the maxilla (Internal view)

Dark blue: Cortical bone, Light blue: Cancellous bone, Green: Implant, Yellow: Abutment, Pink: Prosthesis

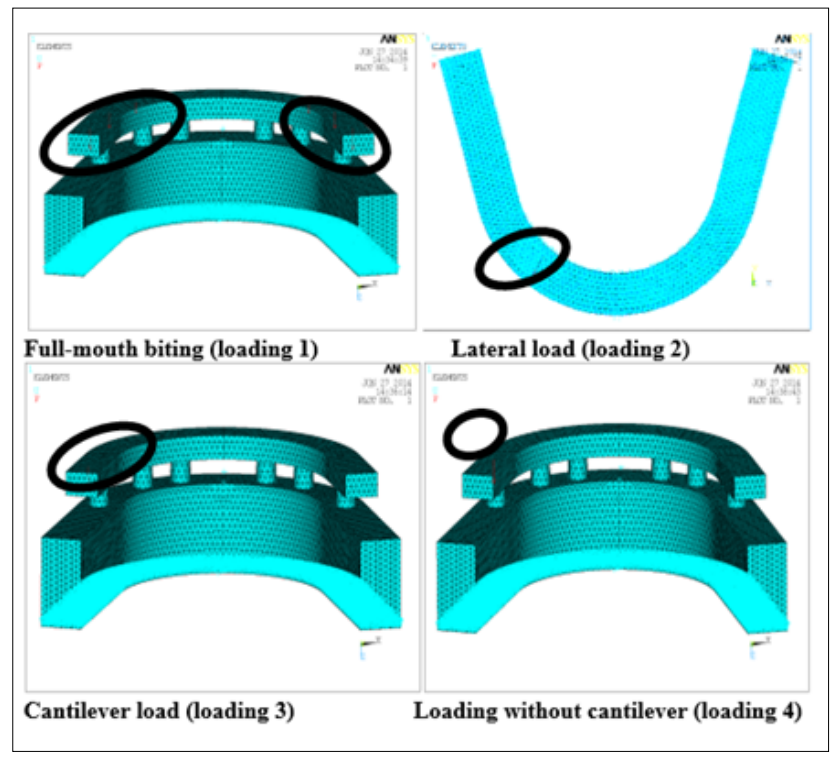

Fig 2.

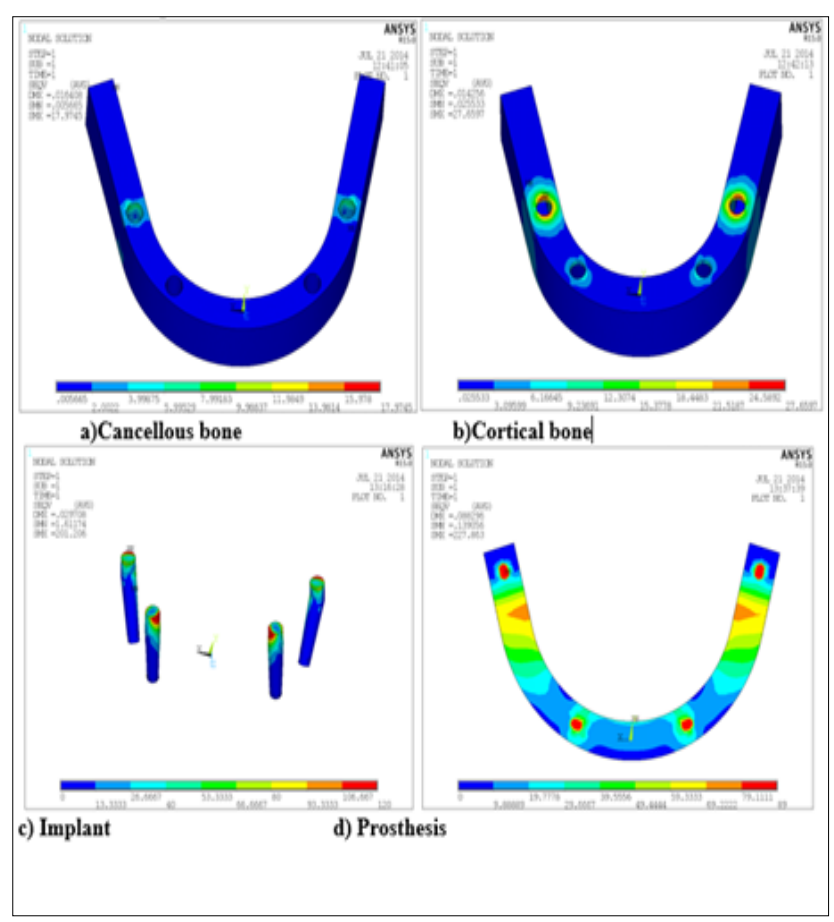

Fig 2 \& 3. Showing Von misses stresses

\section{Discussion}

The von Mises stresses were obtained for all the six models under the desired loading criteria for cancellous bone, cortical bone, implant, prosthesis in the present study.

\section{Stress analysis of cancellous bone:}

In all loading conditions on cancellous bone in all the models, the maximum stress values were located immediately adjacent to the distal implant on the distal side.

On comparison of the maximum von Mises stresses in all models, in loading condition 1 and loading condition 2, model 3 showed least 
stresses (9.4MPa and $5.2 \mathrm{MPa}$ respectively) (Table 3). In both these conditions, model 4 and $6(14.05 \mathrm{MPa}$ and $15.8 \mathrm{MPa}$ respectively) showed 1.5 times greater stress values than model 3 and 5 (9.4MPa and 10.1 MPa respectively) (Table 3). However, these values were still less than the stress values for model 1 and $2(17.9 \mathrm{MPa}$ and 21.1MPa respectively). Hence, during full mouth biting load and lateral load, models with tilted distal implants showed lesser stress values than models with parallelly placed implants. In loading condition 3 (presence of cantilever load), model 6 showed least stress values (9.1MPa) and in loading condition 4 (absence of cantilever load), model 5 showed least stress values (7.8MPa) (Table 3 ) and in both the conditions, models with tilted distal implants showed lesser stress values than models with parallelly placed implants.

The maximum von Mises stresses were less in the models with tilted implants in comparison with the models with parallelly placed implants in all loading conditions on the cancellous bone.

In a study conducted by Giovanni Fazi et al [4], using five models ( 4 parallelly placed, 4 parallelly placed equidistantly, all on four with distal implants tilted at an angle of $17^{\circ}$ and all on four with distal implants tilted at an angle of $34^{\circ}$ and 5 parallelly placed implants) in mandible, he observed fewer stresses in all on four models than parallelly placed models on cancellous bone.

In the present study in the maxilla, similar observations were observed as fewer stresses were observed in model 3 when compared to model 1 on cancellous bone.

In all the models, in cancellous bone, stress values were found to be less in the absence of cantilever when compared to the presence of cantilever. This is in accordance with the study conducted by Guilherme Carvalho Silva et al [5].

Hence, "All-on-four" and "All-on-six" models with distal implants tilted at an angulation of $30^{\circ} \& 45^{\circ}$ showed von Mises stresses less than the models with parallelly placed implants on cancellous bone.

\section{Stress analysis of cortical bone}

In all loading conditions on cortical bone, as in the cancellous bone in all models, the maximum stresses were observed immediately adjacent to the distal side of the distal implant.

On comparison of the maximum von Mises stresses in loading conditions 1, 2, 3, and 4 in cortical bone, model 6 showed most stresses (100MPa,59.8 MPa, $85 \mathrm{MPa}, 58.8 \mathrm{MPa}$ respectively) followed by model $4(90 \mathrm{MPa}, 57.1$ $\mathrm{MPa}, 85 \mathrm{MPa}, 55 \mathrm{MPa}$ respectively). In loading condition $1,3,4$; model 1 with stress values 27.6MPa, 36.3 MPa, 35.4 MPa and model 2 with stress values $33.4 \mathrm{MPa}, 27.7 \mathrm{MPa}, 24.8 \mathrm{MPa}$ were less than or almost equivalent to model 3 with stress values $37.9 \mathrm{MPa}, 40 \mathrm{MPa}, 37.8 \mathrm{MPa}$ and model 5 with stress values $40.6 \mathrm{MPa}, 40$ $\mathrm{MPa}, 31 \mathrm{MPa}$ respectively. In loading condition 2 , model 3 with stress value $19 \mathrm{MPa}$ and model 5 with stress value $20 \mathrm{MPa}$ showed stress values 3 times lesser when compared with all the other models (model 1 - 55.8 MPa, model 2 -56.3 MPa, model 4 - 57.1 MPa, model $6-59.8 \mathrm{MPa}$ ) (Table 4).

On comparison of the maximum von Mises stresses in all models in loading conditions 1 and 2 , model 4 with stress value $90 \mathrm{MPa}$ and model 6 with stress value $100 \mathrm{MPa}$ were found to be 2.5 times greater than model 3 with stress value $37.9 \mathrm{MPa}$ and model 5 with stress value 40.6 MPa (Table 4)

On comparison of the maximum von Mises stresses in all models in loading condition 3, presence of cantilever loading, model 4 and 6 with stress values of $85 \mathrm{MPa}$ showed stresses two times greater than model 3 and 5 with stress values of $40 \mathrm{MPa}$ (Table 4).

On comparison of the maximum von Mises stresses in all models in loading condition 4, absence of cantilever loading, model 4 with stress value of $55 \mathrm{MPa}$ and model 6 with stress value of $58.8 \mathrm{MPa}$ showed stresses 1.5 times greater than model 3 with stress value $37.8 \mathrm{MPa}$ and model 5 with stress value $31 \mathrm{MPa}$ (Table 4) respectively.

In a study conducted by Tasneem Begg et al. [6], analyzing the photoelastic strain patterns surrounding distal implants placed at 0,15,30 and $45^{\circ}$ angles. Observations showed no remarkable difference in strain magnitude between models of implants placed at $0,15,30^{\circ}$, but the increase in strain pattern for $45^{\circ}$ angled implants.

In the present study, similar observations were seen in maximum stress values in cortical bone with stress values found to be similar in model $1,2,3$ and 5 whereas the stress values were found to be increased in model 4 and 6 when compared to model 3 and model 5 respectively. The stress values were observed to be 2.5 times greater (loading 1and 2), 2 times greater (loading 3), 1.5 times greater (loading 4) in model 4 and 6 with an increase of tilt of the distal implant from $30^{\circ}$ to $45^{\circ}$. It can also be noted that stress values were marginally increased from $0^{\circ}$ tilt of implant to $30^{\circ}$ tilt of implant, i.e. in comparison from model 1 and model 3 and also model 2 and model 5.

In a study conducted by Giovanni Fazi et al. [4], 
using five models (4 parallelly placed, 4 parallelly placed equidistantly, all on four with distal implants tilted at an angle of $17^{\circ}$ and all on four with distal implants tilted at an angle of $34^{\circ}$ and 5 parallelly placed implants) in mandible, they observed less stresses in all on four models than parallelly placed models on cortical bone.

In the present study in the maxilla, stresses were slightly greater in model 3 (four implant model with tilted distal implants at an angulation of $30^{\circ}$ ) when compared to model 1 (four parallelly placed implant model) on cortical bone. Hence, models with tilted implants up to $30^{\circ}$ tilt of the implant showed stress values equivalent to models with parallelly placed implants, but the von Mises stresses increased remarkably for models with tilted implants up to $45^{\circ}$ on cortical bone.

In all the models, on cortical bone, stress values were found to be less in the absence of cantilever when compared to the presence of cantilever. This is in accordance with the study conducted by Guilherme Carvalho Silva et al. [5].

"All-on-four" and "All-on-six" models with distal implants tilted at an angulation of $30^{\circ}$ showed a marginal increase in the stress value in presence and absence of cantilever when compared with the parallelly placed implant models in full mouth biting load, but a decrease in the stress value was observed in the presence of lateral load. "All-on-four" and "All-on-six" models with distal implants tilted at an angulation of $45^{\circ}$ showed a remarkable increase in the von Mises stresses in presence and absence of cantilever when compared to all the other implant models and a marginal increase in the stress value was observed in the fact of lateral load.

\section{Stress analysis on the implant}

In loading condition 1, i.e. full mouth biting load, in implant, the von Mises stresses were observed on the mesiopalatal aspect and distal aspect of the distal implant on model 3, 4, 5 and 6 . On comparison of the maximum von Mises stresses Model 4 (90MPa) followed by model 6 (100MPa) (Table 5) showed least stresses and stresses in tilted implant models were equivalent to or less than model 1 and 2. Hence, during full mouth biting load, the maximum stresses in all the models were almost identical.

On comparison of the maximum von Mises stresses in loading condition 2, i.e. lateral load, model 1 (20.6MPa) and model 2 (23.2MPa) (Table 5) showed least stresses when compared to other models. The stresses were observed on the mesiobuccal aspect of the distal implants in all models.

In loading condition 3 , i.e. presence of cantilever, the von Mises stresses were maximum on the mesial and distal aspect of distal implants. On comparison of the maximum von Mises stresses in all models, model $5(80 \mathrm{MPa})$ showed the least stresses equivalent to model 1 and $2(80 \mathrm{MPa})$ (Table 5). The other models, i.e. model 3, 4 and 6 (100MPa, $92 \mathrm{MPa}$ and $92 \mathrm{MPa}$ respectively) (Table 5), showed relatively greater values.

On comparison of the maximum von Mises stresses in all models in loading 4 , i.e. absence of cantilever loading, model 3 (45MPa) (Table 5) showed the least stress values. The von Mises stresses were maximum on the distal aspect of distal implants.

The results obtained in the present $\mathrm{FE}$ analysis showed maximum stress patterns on mesiopalatal area of the tilted implant neck in case of full mouth biting load in model 3, 4, 5 and 6 (tilted implant models) and distal area of distal implants in model 1, 2; mesiobuccal area of the tilted implant neck in case of lateral load in all models; on mesial and distal aspect of distal implants in the presence of cantilever load in all models; distal aspect of the tilted implant neck in the absence of cantilever load in all models.

According to Guilherme Carvalho Silva et al. [5], maximum stress patterns were observed on mesio-palatal area of the tilted implant neck in case of full mouth biting load, mesiobuccal area of the tilted implant neck in case of lateral load, mesio-palatal area of the tilted implant neck in the presence of cantilever load, mesial area of the tilted implant neck in the absence of cantilever load in four implants supported and six implants supported prosthesis with distalmost implants tilted at an angulation of $45^{\circ}$. Maximum stresses were observed to be more in "All-on-four" model when compared to six implant model in all loading conditions.

These results were in accordance with the present study except in the absence of cantilever load where although stresses were observed in the mesial aspect of the distal implants, the maximum stresses were observed in the distal aspect of the distal implants in all models. Maximum stresses in the present study were also observed to be more in model 3 and model 5 when compared to model 4 and model 6 in all loading conditions.

In all the loading conditions in the present study, maximum stress values in the absence of cantilever load were found to be less than in the presence of cantilever load. In model 3, the stress values on the implant were found to be reduced to $55 \%$ and up to $25 \%$ in models 4,5 and 6 . These values were in accordance with a study by Guilherme Carvalho Silva et al. [5], where stress values were found to be reduced 
to $48 \%$ in both four implant-supported, and six implants supported models with tilted distal implants.

In all implant models in the present study, the decrease in stress values were found to be more for tilted implant models (model 3, 4, 5 and 6) in the absence of cantilever than in the presence of cantilever when compared to parallelly placed implant models (model 1 and 2).

In all implant models in the present study, in the presence of lateral load, tilted implant models showed greater stress values when compared to parallel placed implant models.

"All-on-four" and "All-on-six" models with distal implants, tilted at an angulation of 30 and $45^{\circ}$ showed lesser or equivalent von Mises stresses when compared with the distal implants of parallelly placed implant models in full mouth biting load under the presence and absence of cantilever. "All-on-four" and "All-on-six" models at distal implants, tilted at an angulation of 30 and $45^{\circ}$ showed greater von Mises stresses when compared with the distal implants of parallelly placed implant models in the presence of lateral load. "All-on-four" and "All-on-six" models recorded stresses in the absence of cantilever that was 55\% less than the same recorded in the presence of cantilever whereas in the parallelly placed implant models only $25 \%$ decrease of stresses in the absence of cantilever from the presence of cantilever were recorded when compared to "All-on-four" and "All-onsix" models with distal implants, tilted at an angulation of 30 and $45^{\circ}$.

\section{Stress analysis on the prosthesis}

In loading condition 1 , the von Mises stresses were maximum on the cantilever region in all models. On comparison of the maximum von Mises stresses in all models, the least stresses were observed on model 1 and 2 (80MPa) (table $6)$, but the results were comparable to and almost equivalent to model 4,5 and 6 (85MPa, $89 \mathrm{MPa}, 82 \mathrm{MPa}$ respectively) (Table 6). Model 3 showed slightly greater stress value $(100 \mathrm{MPa})$ (Table 6) when compared to all the other models.

In loading condition 2, i.e. lateral load, the von Mises stresses were maximum on the canine region in all models. On comparison of the maximum von Mises stresses in all models, model 3, 4, 5 and 6 showed stress values equivalents to model 1 and 2 .

In loading condition 3, i.e. presence of cantilever loading, the von Mises stresses were maximum on the cantilever region in all models. On comparison of the maximum von Mises stresses in all models, model 3, 4, 5 and 6 showed stress values equivalent to model 1 and 2 .
In loading condition 4, i.e. absence of cantilever loading, the von Mises stresses were maximum on the 2 nd premolar region in all models. On comparison of the maximum von Mises stresses in all models, the least stress values was seen in model 5 (20 MPa) followed by model 3, 4 (22 MPa) (Table 4). Model 3, 4, 5 and 6 showed stress values less than model 1 and 2 .

In all the models, stress values were observed to be decreased by 4 times on the prosthesis in the absence of cantilever when compared to the presence of cantilever. This is in accordance with Guilherme Carvalho Silva et al [5], who observed up to a $90 \%$ decrease in the stress values.

In the present study, "All-on-four" and "Allon-six" models with distal implants tilted at an angulation of 30 and $45^{\circ}$ showed lesser or equivalent von Mises stresses when compared with the parallelly placed implant models. "All-on-four" and "All-on-six" models showed a decrease of stress values by four times from presence of cantilever to absence of cantilever.

Tilting the distal implants for a full-arch fixed prosthesis in maxilla results in shorter cantilever and avoids the close proximity to maxillary sinus whereas in case of placement of straight implants for full-arch fixed prosthesis requires placement of short and wide implants or placement of the distal-most implants in the canine-1st premolar region with long cantilevers or extra surgical procedure like sinus lift(direct or indirect) thereby resulting in increased morbidity and time.

According to Chiara M. Bellini et al. [7], The more distal position of the posterior implant and the resulting shorter cantilever may have a role in the reduction of stress values in the implant.

According to Bevilacqua et.al. [8], tilting of the distal implant by $30^{\circ}$ in an FFP decreased the level of stress by $52 \%$ and $47.6 \%$ in compact bone and cancellous bone respectively, when compared to vertical implants supporting FFP with longer cantilevers.

In the present study, although the distal-most straight implants in the parallelly placed implant models and tilted distal-most implants in the tilted implant models were placed in the 1st premolar area with equal cantilever, models with tilted distal implants showed fewer stresses than models with parallelly placed implants in the cancellous bone. Still, stress values were more in tilted implant models in the cortical bone.

In all the models, in cancellous bone, cortical bone, implant and prosthesis "All-on-four" model with distal implants tilted at an angulation of $30^{\circ}$ showed stress values less than or equivalent 
to all the other models, i.e. All-on-four models with distal implants tilted at an angulation of 45; "All-on-six" models with distal implants tilted at an angulation of $30^{\circ}, 45^{\circ}$; four parallelly placed implant model and six parallelly placed implant model except in implant (loading criteria 3; the presence of cantilever) and prosthesis (loading criteria 1; full mouth biting load) where maximum stresses were observed.

\section{Conclusion}

All-on-four concept with tilted distal implants at an angulation of $30^{\circ}$ showed stress values favourable for the rehabilitation of completely edentulous maxilla, but the presence of cantilever resulted in maximum stresses and remained an area of concern. All-on-four, as the name suggests, requires placement of four implants for complete rehabilitation of maxilla or mandible with favourable stresses, thereby reducing time, morbidity and cost of procedures when compared to six parallelly placed implant model and All-on -six concept.

Conflict of interest : Nil

Source of funding : Nil

\section{REFERENCES}

1. Charles A. Babbush. The All-on-Four Immediate Function Treatment Concept with Nobel Active Implants: A Retrospective Study. Journal of Oral Implantology 2011;37(4):431-45

2. Christopher. Implant rehabilitation in the edentulous jaw: The "All-on-four concept" immediate function. Australian dental practice 2012; 4: 138-48

3. Tommaso Grandi. Immediate Loading of four (All-on-four) Post-extractive implants supporting mandibular cross arch fixed prosthesis - 18 month follow up from a multi centre prospective cohort. Eur J Oral Implantol 2012;5(3):277-85

4. Giovanni Fazi e. Three-Dimensional Finite Element Analysis of Different Implant Configurations for a Mandibular Fixed Prosthesis. Int $\mathrm{J}$ Oral Maxillofac Implants 2011;26:752-9

5. Guilherme Carvalho Silva. Stress patterns on implants in prostheses supported by four or six implants :A three dimensional finite element analysis. Int J Oral Maxillofac Implants 2010,25:239-46

6. Tasneem Begg. Stress patterns around distal angled implants in the All-on-four concept configuration. Int $\mathrm{J}$ Oral Maxillofacial Implants 2009;24:663-71

7. Chiara M. Bellini. Comparison of tilted versus non-tilted implant -supported prosthetic designs for the restoration of the edentulous mandible. A biomechanical study .Int $\mathrm{J}$ oral Maxillofacial implants 2009,24:511-2

8. Bevilacqua $H$. Three dimensional finite element analysis of load transmission using different implant inclinations and cantilever lengths. Int J Prosthodont 2008;21:539-42 